\title{
CHROMOSPHERIC VARIATION \\ IN COOL SUPERGIANT STARS
}

\author{
J. A. EATON AND G. W. HENRY \\ Tennessee State University \\ Nashville, TN, USA
}

\begin{abstract}
Stars close to the main sequence, if they rotate rapidly enough, show magnetic activity in the form of spots, active regions, and strong chromospheres. With the exception of the hybrid stars, stars cooler and more luminous than the so-called Linsky-Haisch dividing line, in contrast, are not known to have any of these phenomena. In fact, it is not clear such stars, even though rotating and convective, show any magnetic phenomena at all. We discuss (1) photometry used to search for spots in the hybrid stars, which we have not found, and (2) evidence for chromospheric variation in $\mathrm{K}$ supergiants that takes the form of enhanced winds, but which may be analogous to the activity of stars closer to the main sequence.
\end{abstract}

\section{Introduction}

Stars near the main sequence (specifically, luminosity classes V, IV, and-to some extent-III) that rotate rapidly enough have solar-like active regions above their surfaces (see, e.g., Noyes et al. 1984, Oranje 1986; Basri 1987). These stars' activity can be understood as the manifestation of magnetism generated by a dynamo operating in or just under the stellar convective zone. Linsky and Haisch (1979), however, found this activity does not characterize the cooler and/or more luminous convective stars (discussed further by Haisch 1987). Instead, the Hertzsprung-Russell diagram is divided into two vast zones. Those stars near the main sequence, tending to be hotter and to have higher surface pressures, show outer atmospheres like the Sun's. They have discrete active regions, coronae, and detectable starspots. In contrast, those giant stars as cool as K4 III and the G-K-M supergiants, which we like to call the windy giants, show no hot transition-region or 
coronal emission; instead, they have mass loss in prominent winds many orders of magnitude greater than solar mass loss.

The effect of rotation and effective temperature in the Sun-like stars is well understood through stellar dynamos. But what are the effects of magnetism and dynamos in these larger windy stars? Such stars are deeply convective and rotating, although slowly in terms of surface speeds. Presumably dynamos would operate in them at some level. In fact, at least the warmer of these objects are thought to have surface magnetic fields, for although magnetism may be unnecessary to drive winds in the cooler windy giants, the best models of winds in $\mathrm{G}$ and $\mathrm{K}$ supergiants depend on magnetic fields to support Alfven waves (Hartmann and McGregor 1980; Holzer and McGregor 1985). Furthermore, some windy stars (the chromospheric hybrids; Hartmann et al. 1980) seem to show actual active regions.

So we should like to begin exploring the possibility of variable magnetic effects in these cool giants and supergiants. How magnetism would manifest itself in these luminous stars is not as obvious as in the active dwarfs, since we already know some aspects of the solar analogy do not apply, so our attempt must necessarily be exploratory and somewhat speculative. In this effort we will concentrate on two questions: (1) are the chromospheric hybrid stars spotted? and (2) are there magnetic (chromospheric) cycles in the $\mathrm{K}$ supergiants?

\section{Chromospheric hybrid stars}

These stars are giants and supergiants that show both the large mass loss of windy giants and the $\sim 10^{5} \mathrm{~K}$ emission formed in transition regions of dwarf stars (Hartmann et al. 1980; Reimers 1982). Since they are prime candidates for having solar-type active regions in conjunction with their massive winds, we might expect to detect starspots on their surfaces. If such stars were indeed found to be spotted, we would then be able to use light curves to measure their rotational periods, and we certainly could relate them to the dwarf stars through dynamos.

To determine whether any hybrid stars may be spotted, we have observed five of them for the past six years with the Vanderbilt/TSU robotic telescope. These stars are listed in Table 1. Inasmuch as all of them are very bright, it has been difficult to observe them reliably with that 16 -inch telescope. Changes in the photometric system, particularly in the early years of this observing program, are difficult to calibrate. Nevertheless, we can say that all five stars have been constant to $\leq 1 \%$ during the past three years, during which the photometric system has been especially stable. The expected precision of the individual nightly mean magnitude differences in this photometry are of the order of $0.004 \mathrm{mag}$ (Henry 1995), while the 
TABLE 1. Hybrid Stars

\begin{tabular}{lccl}
\hline Star & $V$ & Type & Notes \\
\hline$\delta$ And & 3.27 & K3 III & binary \\
$\gamma$ Aql & 2.72 & K3 II & binary \\
$\iota$ Aur & 2.69 & K3 II & \\
$\theta$ Her & 3.86 & K1 IIa & \\
$\sigma$ Oph & 4.34 & K2 II & \\
\hline
\end{tabular}

actual measured standard deviations of nightly means with respect to seasonal averages were $0.0050 \mathrm{mag}$. The largest season-to-season variation in the data after JD 2,449,117 was 0.004 mag (vs. a median change of 0.002 mag). Figure 1 gives an example of the photometry. We see no convincing evidence in these data for variation from dark spots with periods of a few hundred days, or less, and amplitudes greater than a few tenths of a percent. These stars thus seem to behave less like the giants among the RS CVn binaries but more like those less active G-K giants observed by Choi et al. (1995), eleven of which showed light constant to $\leq 1 \%$ in spite of $\mathrm{Ca}$ II $\mathrm{H}$ and $\mathrm{K}$ variation.

\section{3. $\mathbf{H} \alpha$ variations in $\mathrm{K}$ supergiants}

In cool giants and supergiants, the Balmer lines are a direct consequence of a chromosphere (Cram and Mullan 1985). These chromospheres seem to be ubiquitous, simply because all $\mathrm{K}$ and early $\mathrm{M}$ giants have roughly the same $\mathrm{H} \alpha$ absorption (e.g., Eaton 1995). Furthermore, in the supergiants, the $\mathrm{H} \alpha$ is so optically thick that its core is formed in the accelerating base of the wind, giving an asymmetric line like that seen in Figure 2 (Mallik $1987,1993) . \mathrm{H} \alpha$ averages over the whole inner chromosphere of cool giants, and may be thought to give a measure of the state of the chromosphere.

We have observed the eight cool supergiants listed in Table 2 over the past several years to search for changes in $\mathrm{H} \alpha$ reflecting changes in chromospheric structure. Initially, this was a project to look for phase dependence of chromospheres of $\zeta$ Aur binaries, but the effects seen in such binaries were not obviously phase dependent, nor did they differ from changes in other stars without hot companions. We might well expect to see variations of $\mathrm{H} \alpha$ in these stars, since Pop. II red giants have shown changes in their $\mathrm{H} \alpha$ profiles (see Smith and Dupree 1988). Figure 3 gives examples of the variation we have detected.

There seem to have been subtle changes throughout the $\mathrm{H} \alpha$ profiles of 


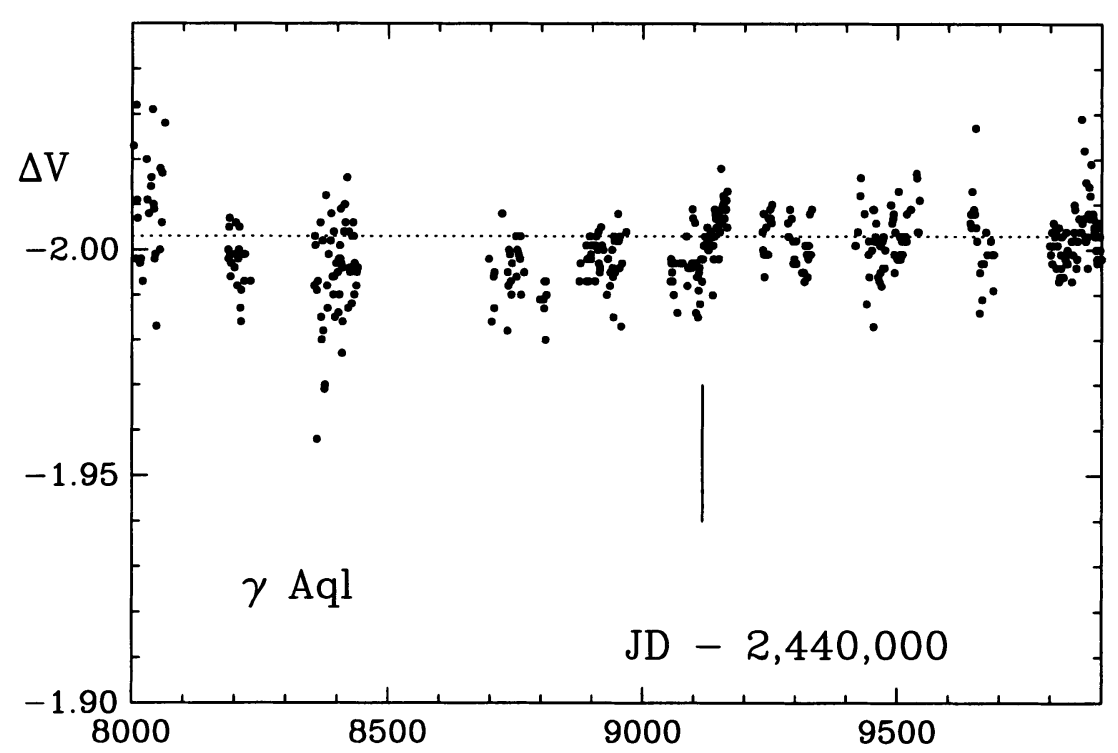

Figure 1. The light variation of $\gamma$ Aql vs. a magnitude difference of -2.003 mag (horizontal dotted line). These observations are constant to within $1 \%$, especially those data taken after JD 2,448,700 with the new TSU precision photometer, even without allowance for changes in the photometric system. The biggest uncertainty has been the dead-time correction applied for stars as bright as the typical hybrid giant. Before date 8700 , it is poorly known; after 8700 but before 9117 it was known moderately well but was rather large; while after 9117 it has been both much lower and better determined. The shift in the data at 9117 (at the solid vertical line) reflects this change in dead time.

TABLE 2. Stars observed at $\mathrm{H} \alpha$

\begin{tabular}{lcccl}
\hline Star & $V$ & Type & P(orb.) & Notes \\
\hline$\zeta$ Aur & 3.75 & K4-5 Ib+B6 & 972 & $\zeta$ Aur binary \\
31 Cyg & 3.79 & K4 Ib+B3-4 & 3784 & $\zeta$ Aur binary \\
32 Cyg & 3.98 & K4-5 Ib+B6-7 & 1147 & $\zeta$ Aur binary \\
22 Vul & 5.15 & G4 I+B7 & 250 & $\zeta$ Aur binary \\
47 Cyg & 4.61 & K2 Ib+B3 V & $\ldots$ & \\
63 Cyg & 4.55 & K4 Ib-IIa & $\ldots$ & \\
$\xi$ Cyg & 3.72 & K4-5 Ib-II & $\ldots$ & \\
$\epsilon$ Peg & 2.39 & K2 Ib & $\ldots$ & \\
\hline
\end{tabular}

all these stars, but the strongest effects are in the blue wings, those parts of the profiles most suceptible to effects of stellar winds. Figure 3 shows three profiles, the dashed curve is typical of $\mathrm{H} \alpha$ in $\mathrm{K}$ giants-neither absorption nor emission. The dot-dashed profile shows blue wing emission, seen in 


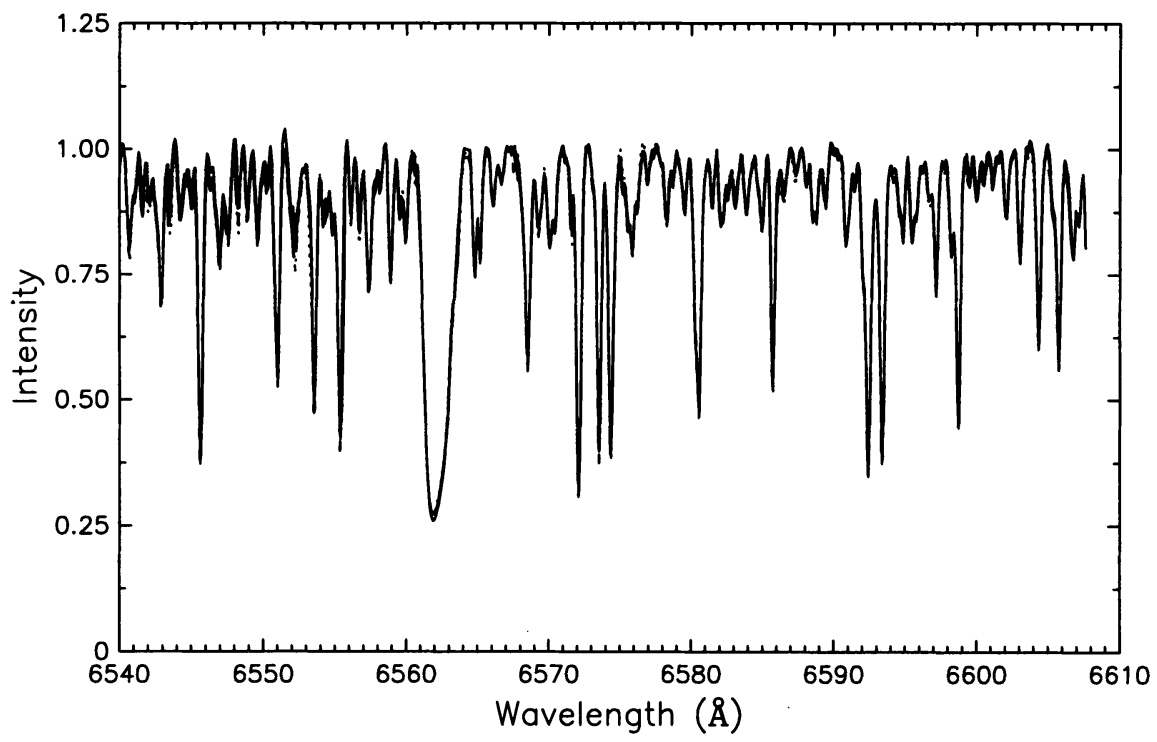

Figure 2. Typical spectrum of the $\mathrm{H} \alpha$ region in a $\mathrm{K}$ supergiant (six spectra of $31 \mathrm{Cyg}$ plotted together). Note how the line core is displaced to the blue and the strength of the line.

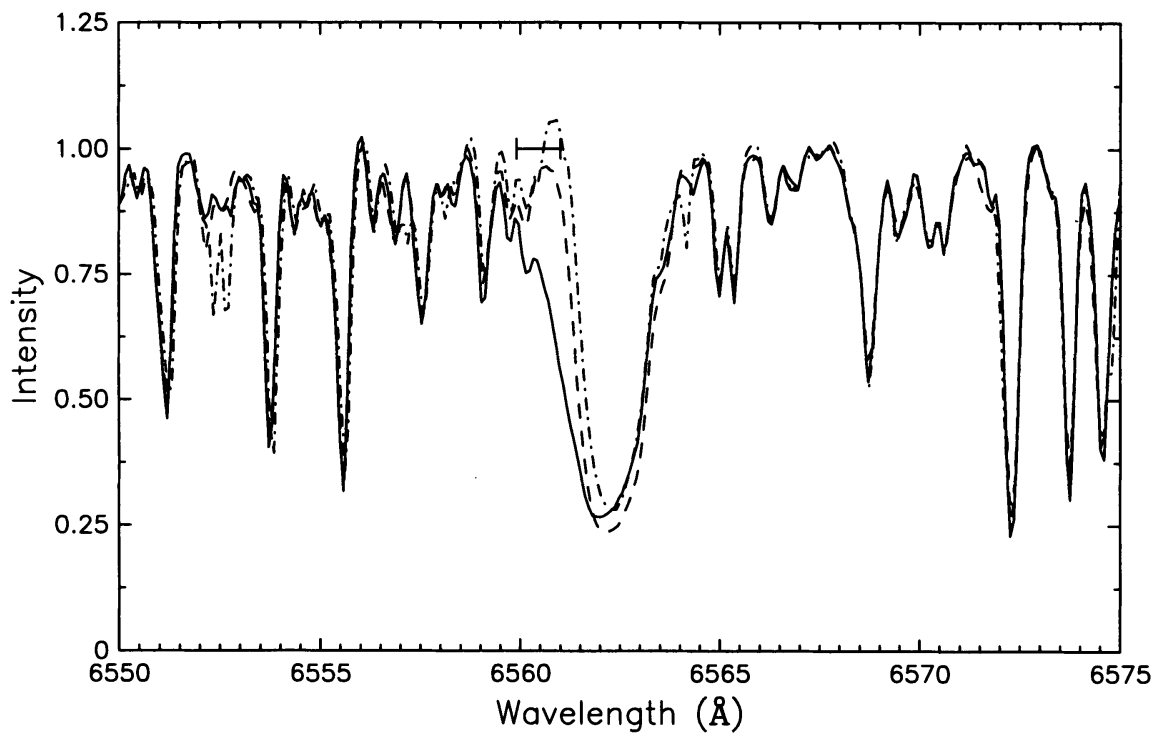

Figure 3. Three H $\alpha$ profiles for $\xi$ Cyg. Changes are especially notable for the blue wing. The same phenomenon was illustrated by Smith and Dupree (1988), Fig. 6. The bar above the profile indicates the part of the spectrum measured to give equivalent widths plotted in Figure 4. 
many supergiants. The solid curve shows excess absorption, which we have detected in all six of our program stars.
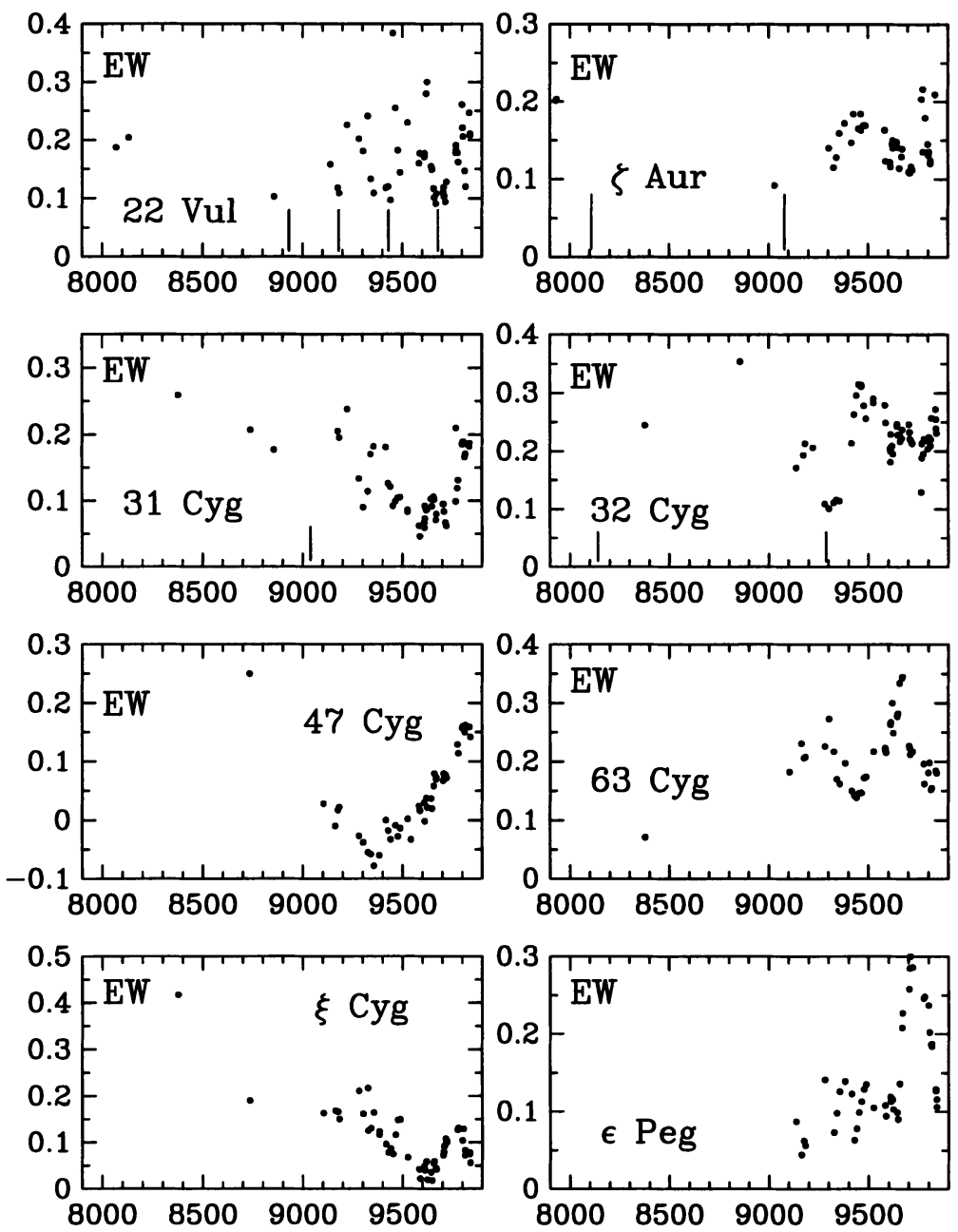

JD $-2,440,000$

Figure 4. Variation of the equivalent width of the blue wing and of $\mathrm{H} \alpha$ in eight cool supergiants. Vertical lines at the bottom of the figures show times of conjunction in $\zeta$ Aur with the $\mathrm{K}$ star in front. Illumination by the B stars in these binaries does not seem to have had much effect on the $\mathrm{H} \alpha$ profiles.

To measure the changes in the $\mathrm{H} \alpha$ profile quantitatively, we have measured the equivalent width in a 1.1- $\AA$ band centered in the blue wing (shown in Figure 3). We plot the time series of these measures in Figure 4. You can see that every one of these stars is variable in the blue wing of $\mathrm{H} \alpha$. Pre- 
sumably the excess absorption represents intensification of the mass loss, in which a denser wind becomes optically thick at a higher, faster level. This is expected from Mallik's calculations. Blue-shifted emission can also come from such a layer, provided the gas is somewhat hotter and more optically thick than in the typical chromospheric model. All of these stars, except perhaps $47 \mathrm{Cyg}$, may show episodic ejections of matter. Especially impressive is the effect in $22 \mathrm{Vul}, 63 \mathrm{Cyg}$, and $\epsilon \mathrm{Peg} ; 31 \mathrm{Cyg}$ and $32 \mathrm{Cyg}$ seem to show strong ejections too. The stars $47 \mathrm{Cyg}$ and $\xi$ Cyg seem to have slow changes in $\mathrm{H} \alpha$ strength that may represent cycles in the wind strength.

The close binary $22 \mathrm{Vul}$ is particularly interesting in that it is the fastest rotating of these supergiants ( $\mathrm{P}=250$ days in a synchronous binary) and it has the strongest activity.

\section{Summary}

We have used two tracers of magnetic fields to begin assessing activity in the windy giants. We find no evidence for extensive spottedness in the chromospheric hybrid stars, which are actually thought to have active regions of some sort. On the other hand, the G and $\mathrm{K}$ supergiants show episodic, possibly cyclic changes in the wind component of $\mathrm{H} \alpha$ which may be the signature of magnetism in such luminous stars.

\section{References}

Basri, G. (1987) ApJ, 316, 377

Choi, H.-J., Soon, W.H., Donahue, R.A., Baliunas, S.L., Henry, G.W. 1995, PASP, 107, 744

Cram, L.E., Mullan, D.J. (1985) $A p J$, 294, 626

Eaton, J.A. (1995) $A J, \mathbf{1 0 9}, 1797$

Haisch, B.M. (1987) Cool Stars, Stellar Systems, and the Sun, No. 5, ed. J.L. Linsky and R.E. Stencel, p. 269

Hartmann, L., Dupree, A.K., Raymond, J.C. (1980) ApJ, 236, L143

Hartmann, L., McGregor, K.B. (1980) ApJ, 242, 260

Henry, G.W. (1995) Robotic Telescopes: Current Capabilities, Present Developments, and Future Prospects for Automated Astronomy, ed. G.W. Henry and J.A. Eaton, ASP Conf. Ser. vol. 89 , p. 44

Holzer, T.E., McGregor, K.B. (1985) Mass Loss from Red Giant Stars, ed. M. Morris and B. Zuckerman, p. 229

Linsky, J.L, Haisch, B.M. (1979) ApJ, 229, L27

Mallik, S.V. (1987) Cool Stars, Stellar Systems, and the Sun, No. 5, ed. J.L. Linsky and R.E. Stencel, p. 319

Mallik, S.V. (1993) ApJ, 402, 303

Noyes, R.W, Hartmann, L., Baliunas, S.L., Duncan, D.K., Vaughan, A.H. (1984) ApJ, 279, 763

Oranje, B.J. (1986) $A \& A, 154,185$

Reimers D. (1982) $A \mathscr{G} A, 107,292$

Smith, G.H., Dupree, A.K. (1988) $A J, 95,1547$ 


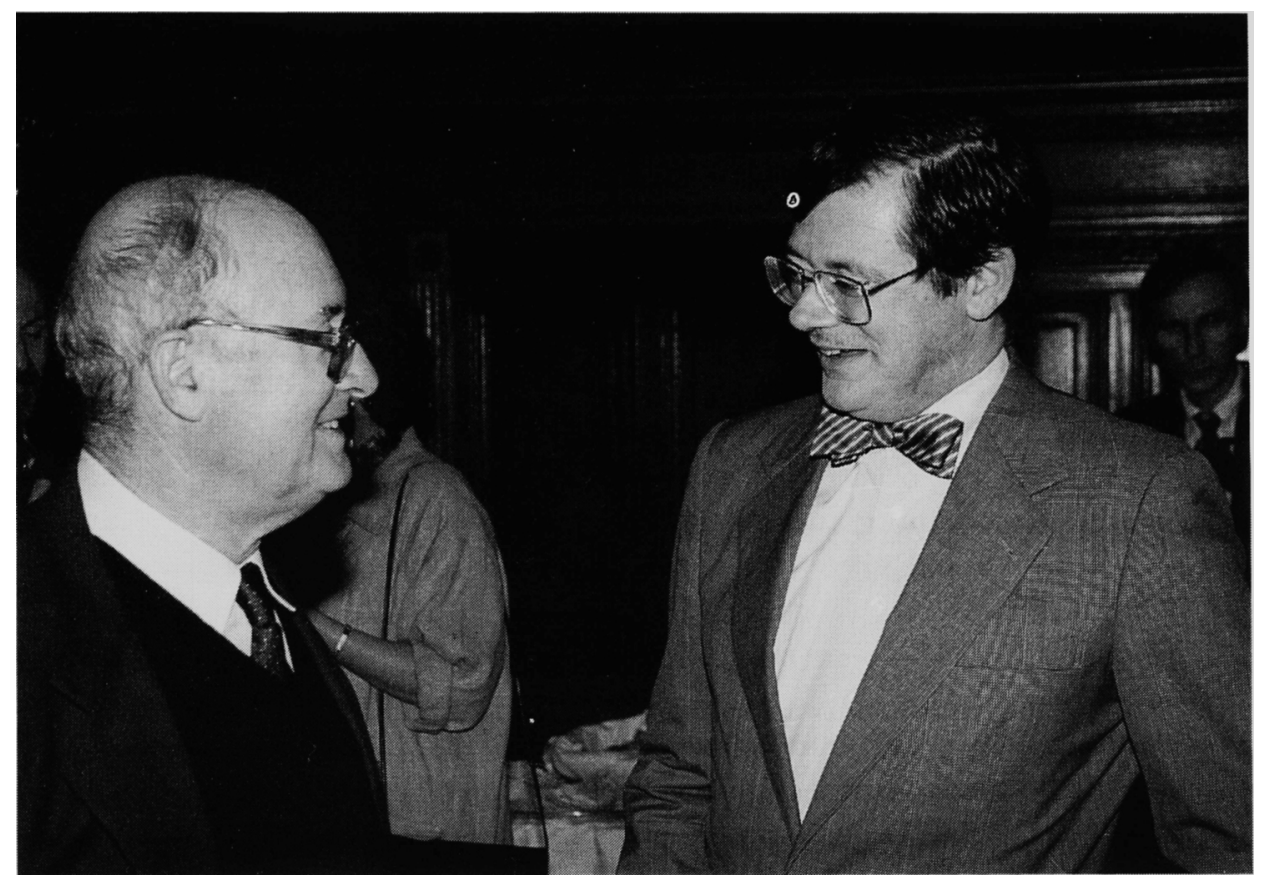

It looks like Joel Eaton (right) is telling a joke to Raphael Steinitz. No kidding.

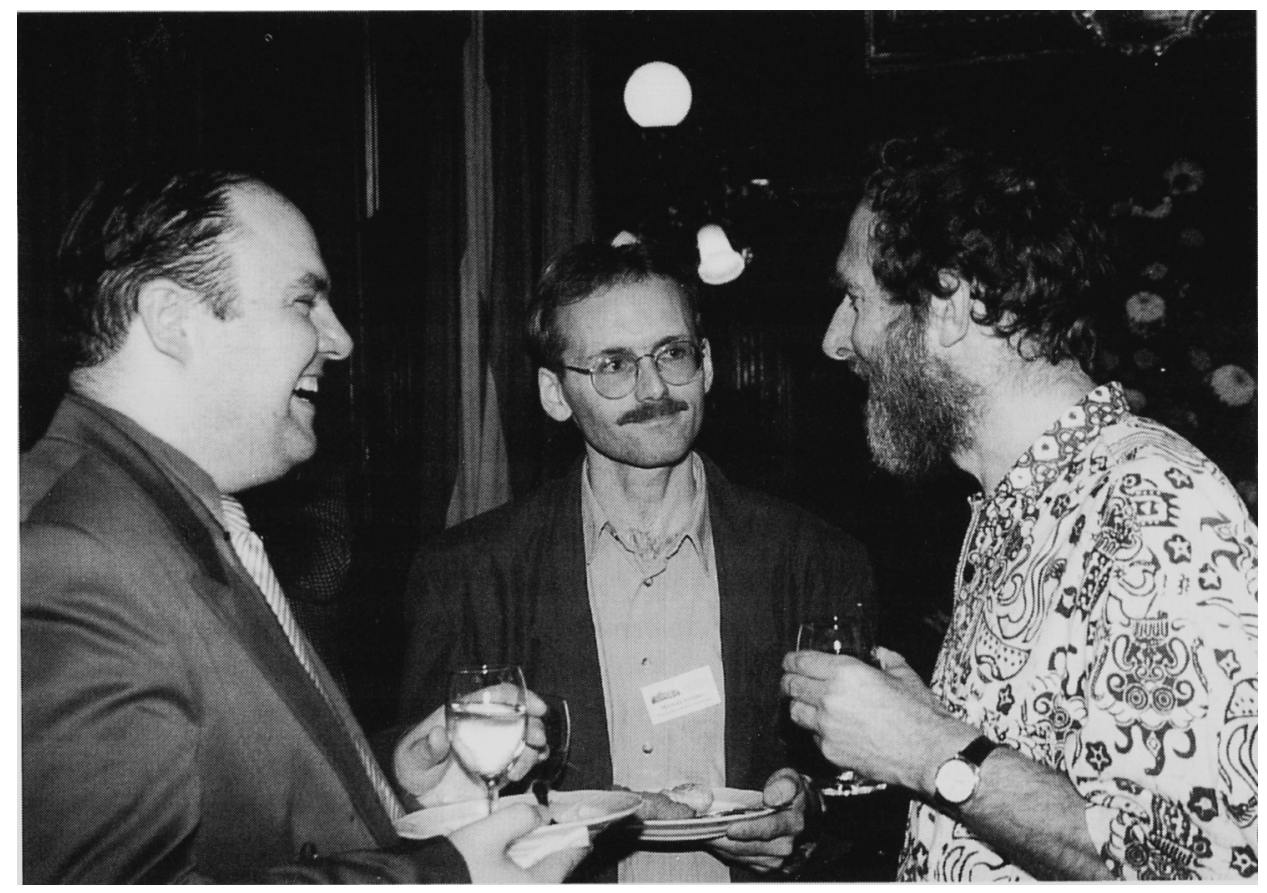

Barry Kellett, Manuel Güdel, and John Butler. 